\title{
A Method for Segmentation of CT Head Images
}

\author{
Sven Lončarić and Domagoj Kovačević \\ Department of Electronic Systems and Information Processing \\ Faculty of Electrical Engineering and Computing \\ University of Zagreb \\ Unska 3, 10000 Zagreb, Croatia
}

\begin{abstract}
A novel method for automatic segmentation of computed tomography (CT) head images of patients having spontaneous intracerebral brain hemorrhage has been presented in this work. The method consists of four major phases. The first phase performs a brightness normalization of a CT image by applying a K-means clustering algorithm to the pixel brightness values. A feature extraction based on a special receptive field is done in the second phase. The third phase performs a pixel classification by means of a feed-forward error-back propagation neural network. In the last phase, a rule-based expert system is used to perform image labeling. The proposed method has been applied to real patient CT images and has shown encouraging results.
\end{abstract}

\section{Introduction}

Image segmentation is an important step in computer-based medical systems for image measurements. The problem of brain image segmentation has been frequently addressed in literature in recent years $[1,2]$. The methods used for segmentation typically utilize advanced artificial intelligence and pattern recognition techniques such as those based on neural networks [3], morphological processing [4], and clustering algorithms [5]. The techniques can also be divided to operator assisted (semi-automatic) and fully automatic methods.

A new approach to automatic segmentation of computed tomography (CT) head images of spontaneous intracerebral brain hemorrhage is presented in this paper. The method utilizes K-means clustering for image brightness normalization, a special retina-like receptive field for feature extraction, a neural network for classification, and an expert-system for region labeling.

The paper is organized as follows. The proposed method is presented in Section 2. Segmentation results are described in Section 3. A conclusion is provided in Section 4.

\section{A Method for CT Head Image Segmentation}

The proposed method performs automatic segmentation of CT head images of human spontaneous intracerebral brain hemorrhage (ICH). The image is divided into five regions of interest: background, skull, brain, ICH, and calcifications. The 
segmentation procedure has four major steps. In the preprocessing step, K-means clustering of the brightness values is used to determine cluster centers which are then moved to predefined positions. In the second step, a receptive field is used for image feature extraction. In the third step, the image is classified using a neural network. Finally, the image regions are labeled using an expert system to produce the final segmented image.

\subsection{Preprocessing}

The histogram of the original CT image has three modes. These modes represent three clusters in the brightness space. A typical CT image and its histogram are shown in Figure 1. If the image is darker or brighter then the three histogram

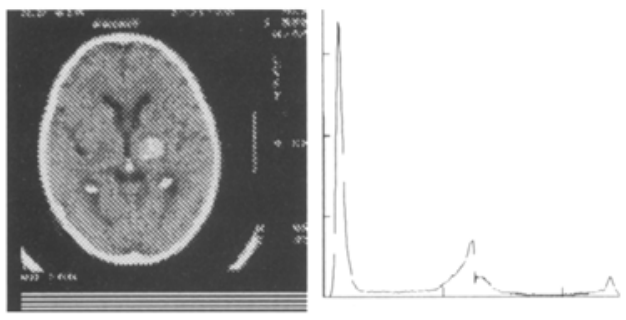

Fig. 1. A typical CT image and its tri-modal histogram.

modes (clusters) are shifted to lower or higher values, respectively. The image brightness can be normalized by moving the histogram modes to a fixed, predefined position.

The image brightness normalization algorithm is described below. In the first step, the cluster centers are determined using a $\mathrm{K}$-means clustering algorithm with three clusters. The Euclidean distance has been used as a distance measure. In the second step, a gray scale transformation is applied to the image. The transformation translates all points in a cluster so that the cluster centroid is translated to a desired location. Let $x_{i}, i=1,2,3$ be the cluster centers of the original CT image. The gray scale transformation is done as follows:

for each pixel $p$ in the image do

for $i=1, \ldots, 3$ do

if pixel $p$ is in cluster $i$

then $b(p)=b(p)+y_{i}-x_{i}$

where $b(p)$ is the brightness value of the pixel $p, p \in N^{2}$, and $y_{i}$ are the predefined (desired) cluster centers.

\subsection{A receptive field for feature extraction}

A special receptive field for image feature extraction has been utilized. The receptive field is used to form a feature vector which is used as an input to the 
neural network classification stage. The receptive field is moved across the image producing a feature vector for each position in the image. The motivation for the topology of the receptive field was found in the human visual system. The density of the rods and cones in the human retina is highest in the center of the field of view and falls off towards the peripheral parts of the retina. The receptive field has been constructed in a similar way.

The center of the receptive field is positioned on the pixel to be classified. The central pixel as well as pixels in some neighborhood are candidates for feature extraction at this particular location. A neighborhood of the circular form has been used in this work.

A proper classification of a pixel requires the information from a larger neighborhood of the pixel. Experiments demonstrated that the minimum neighborhood radius is about 30 pixels for images of size $512 \times 512$. The use of all pixels in such a large neighborhood would result in a feature vector having several thousand elements. To avoid this problem a special receptive field has been designed. The receptive field utilizes a selected subset of points in a circular neighborhood. This subset is defined by point coordinates relative to the pixel which is being classified. The features are extracted only at locations determined by the elements of the receptive field. The receptive field consists of receptive rings. A receptive ring is described by its radius and grid. The coordinates of the $i$-th receptive ring are equidistantly located on the circle of radius $r_{i}$ and are separated by the angle $\phi, \phi=2 \pi / N_{i}$ where $N_{i}$ is the number of elements of the $i$-th receptive ring. An example of the receptive field is shown in Figure 2. The rings

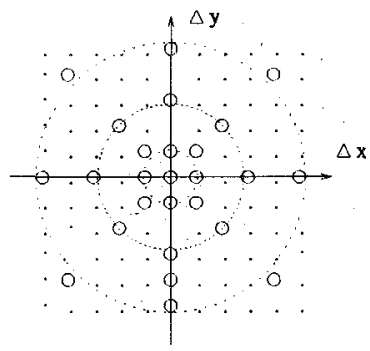

Fig. 2. A receptive field with 4 receptive rings with radius equal to $0,1,3$, and 5 pixels.

with larger radius have larger distance between points due to a constant central angle between elements on a ring. This is justified by the smaller significance of the peripheral (more distant) neighboring pixels.

The actual feature values are the mean and the standard deviation of the brightness of pixels contained in a receptive ring. In such a way the total number of features is equal to two times the number of receptive rings. The dimensionality of this feature vector is much smaller then if all pixel values were used. Experiments have shown no degradation in segmentation accuracy using this lower dimensionality feature vector. 


\subsection{Neural Network Classification}

A feed-forward neural network has been used for CT classification. The error back-propagation learning has been utilized for network training. The classification is performed as follows. For each pixel in the image features are extracted. Features are then fed to the input layer of the neural network. A pixel is assigned to the class which is represented by the output neuron with highest activation.

Several experiments have been made to determine the optimal network topology. A network having one hidden layer required a large number of neurons resulting in a slow training. The best results were obtained using a neural network having two hidden layers. For each ring in the receptive field the input layer has two neurons: one for the mean and one for the standard deviation. The total number of input neurons depends on the number of rings in the receptive field. The output layer has five neurons corresponding to the five regions of interest.This means that for each position in the image exactly one output neuron must activate to indicate the classified region.

\subsection{Expert System Labeling}

The classification performed by the neural network is not perfect. The regions of interest are broken into smaller regions and it is necessary to perform the labeling of the smaller regions so that the actual regions of interest can be formed. The solution to the labeling problem is found using a rule-based expert system.

A rule-based expert system represents knowledge in the form of logical rules. The facts contain relevant information about the problem. The rules (the knowledge) are applied to the facts (the problem) to derive the conclusion (the solution). In this case, the facts consist of region information such as the region size, neighbors, and class to which it was classified by the neural network. The rules are based on two assumptions: the assumption about the minimum region size and the assumption about the possible region neighbors. A graph showing the neighborhood relations between the regions of a CT image is shown in Figure 3. A neighborhood relation between the brain and the background regions exists for the following two reasons. First, in CT scans taken at the level of the patient's eye there may be a gap within the skull region. Second, there are images in which the head is not properly centered (a part of the skull is missing) and the brain is touching the background. The hemorrhage region can only be neighbor to the brain region which is a limiting factor. This limitation is imposed to eliminate situations where the hemorrhage could be a neighbor to the skull. In such a case both regions would have a similar brightness and could be confused. It seems, however, that this kind of situation is not likely to happen, in fact in the CT image database which was used in this work there were no images with the skull neighboring the hemorrhage region. Another assumption made on the regions is that there is a lower bound on the region size as shown in Table 2. 


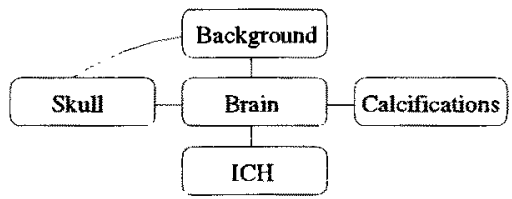

Fig. 3. A model of region neighborhood of a CT image.

\section{Experimental Results}

\subsection{Preprocessing}

A K-means algorithm with three clusters has been used to find the actual cluster centers of the pixel brightness values. The values of the desired cluster centers are set to the cluster centers of the medium bright image. These values are equal to 19, 118, and 235, respectively. An example of a CT image before and after brightness normalization is shown in Figure 4.
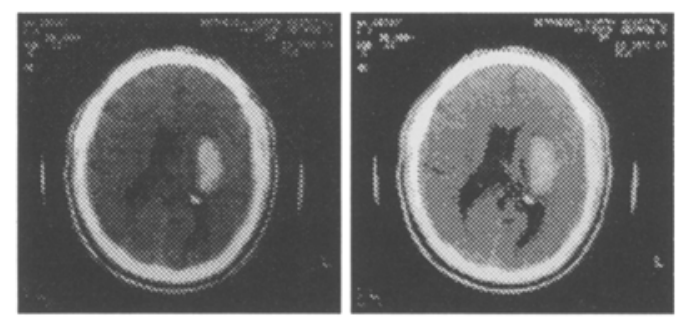

Fig. 4. A dark CT image before and after brightness normalization.

\subsection{Receptive Field Structure}

Experiments have been made to determine the optimal size and shape of the receptive field. For each receptive field topology a classification performance of the receptive field and the neural network was tested by measuring classification accuracy. The accuracy is measured as the ratio of correctly classified image points to the total number of image points. Some receptive field topologies with classification accuracies are listed in Table 1. The first column in the table shows the number of rings in the receptive field. The second column shows radius for each ring, in pixels. The third column shows the total number of receptive field points. The fourth column shows the relative segmentation accuracy. Note that a ring with radius equal to zero has only one element. It is obvious from Table 1 that the receptive field with seven rings represents the optimal choice. Larger receptive fields do not show any increase is classification accuracy. The features used for describing the pixels are the mean and the standard deviation and they are calculated for each ring separately. This makes the method rotation invariant. 


\begin{tabular}{|c|l|c|c|}
\hline Rings & Radius & Elements & Classification accuracy \% \\
\hline 4 & $0,1,8,30$ & 25 & 93.6 \\
\hline 5 & $0,1,4,12,30$ & 33 & 96.3 \\
\hline 6 & $0,1,4,12,30,40$ & 41 & 96.6 \\
\hline 7 & $0,1,3,7,14,30,40$ & 49 & 98.7 \\
\hline 8 & $0,1,2,6,15,23,33,45$ & 57 & 98.6 \\
\hline 9 & $0,1,2,4,7,15,24,34,45$ & 65 & 98.7 \\
\hline
\end{tabular}

Table 1. Different receptive field topologies and their classification accuracy.

\subsection{Classification}

The results shown here are obtained using a receptive field having seven rings with eight points per ring. Each ring is characterized using the mean and the standard deviation of the eight pixels contained in the ring. Feature vectors produced in such a manner have fourteen elements. Therefore, the number of neurons in the input layer is equal to fourteen. Two hidden layers were used with twelve and eight neurons, respectively. The output layer has five output neurons corresponding to the five regions of interest: brain, skull, hemorrhage, background, and calcifications.

The neural network was trained using the Stuttgart Neural Network Simulator V4.1. The error-backpropagation learning paradigm was used. Three different images were used for training. Each image was randomly sampled at 2500 locations (500 per region) and the obtained data was used as a training set. An example of original and classified image is shown in Figure 5. It is evident that a
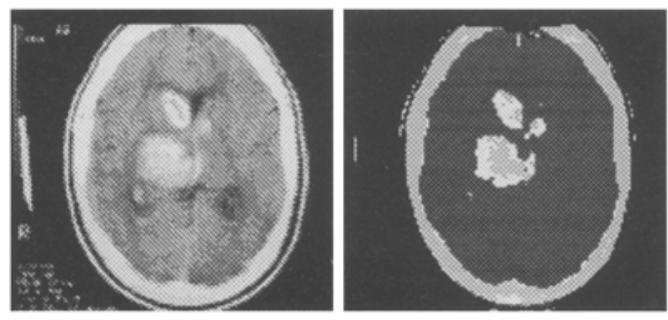

Fig. 5. CT images (top row) and classified images (bottom row).

number of small regions appeared in the classified images due to neural network behavior. For this reason a consequent labeling stage is necessary.

\subsection{Labeling}

The rule-based expert system was used for region labeling as explained in Section 2.4. The software was implemented in C language. 
The minimum region areas are listed in Table 2 and are expressed relative to the CT image area. These relative areas are used for the expert system rules. The results of the expert system for region labeling are shown in Figure 6.

\begin{tabular}{||c|r|}
\hline Region & Region Area in \% \\
\hline background & 8.0 \\
\hline skull & 1.5 \\
\hline brain & 10.0 \\
\hline ICH & 0.2 \\
\hline calcifications & 0.05 \\
\hline
\end{tabular}

Table 2. Minimal allowed region areas relative to the CT image area.
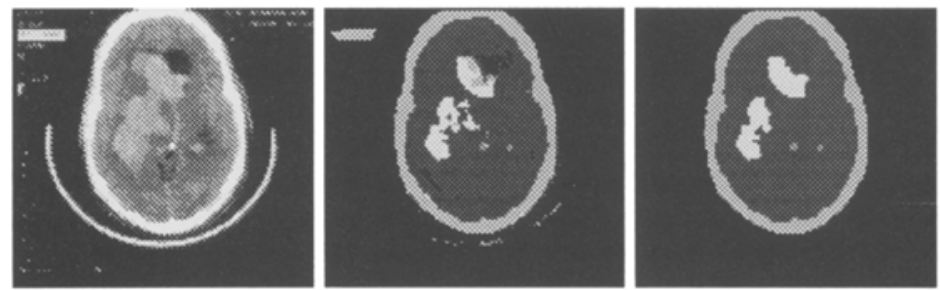

Fig. 6. From left to right: original CT image, image classified using neural network, and CT image labeled using the expert system.

\subsection{Discussion}

A confusion (or discrimination) matrix obtained by averaging segmentation results for ten images is shown in Table 3. The numbers in the table represent the percentage of correctly classified pixels in the image with respect to the number of pixels in a region. It can be seen from the confusion matrix that there is no confusion between classes which cannot be neighbors as defined in rules of the expert system. The largest misclassification rate is evident for the hemorrhage region being classified as the brain region in $5.3 \%$ of the hemorrhage region pixels.

\section{Conclusion}

In this work a novel method for automatic segmentation of CT head images of patients having spontaneous intracerebral brain hemorrhage has been presented. The method consists of four phases. The first phase includes normalization of a 


\begin{tabular}{|c|c|c|c|c|c|}
\hline region/segmented as & background & skull & brain & calcifications & ICH \\
\hline background & 99.64 & 0.36 & 0 & 0 & 0 \\
\hline skull & 1.47 & 98.27 & 0.26 & 0 & 0 \\
\hline brain & 0 & 0.55 & 97.20 & 0.14 & 2.1 \\
\hline calcifications & 0 & 0 & 3.11 & 96.43 & 0.46 \\
\hline ICH & 0 & 0 & 5.30 & 0 & 94.70 \\
\hline
\end{tabular}

Table 3. Confusion matrix.

$\mathrm{CT}$ image histogram. Feature extraction using a special receptive field motivated by the human visual system is done in the second phase. The third phase performs pixel classification by means of the feed-forward error-back propagation neural network. In the last phase, an expert system is used to perform image labeling. The expert system knowledge is based on assumptions on region sizes and region neighborhood relations. The proposed method has been applied to real $\mathrm{CT}$ images and has shown encouraging results.

\section{References}

1. J. C. Bezdek, L. O. Hall, and L. P. Clarke. Review of MR image segmentation techniques using pattern recognition. Medical Physics, 20:1033-1048, 1993.

2. S. Loncaric, A. P. Dhawan, T. Brott, and J. Broderick. 3-D image analysis of intracerebral brain hemorrhage. Computer Methods and Programs in Biomedicine, 46:207-216, 1995.

3. G. I. Chiou and Jeng-Neng Hwang. A neural network-based stochastic active model (NNS-SNAKE) for contour finding of distinct features. IEEE Transactions on Image Processing, 4:1407-1416, 1995.

4. J. G. Thomas, R. A. Petters, and P. Jeanty. Automatic segmentation of ultrasound images using morphological operators. IEEE Transactions on Medical Imaging, 10:180-185, 1991.

5. S. Loncaric, D. Cosic, and A. P. Dhawan. Segmentation of CT head images. In Proceedings of the 10th International Symposium Computer Assisted Radiology, page 1012. Elsevier, Amsterdam, 1996. 